\title{
Reliability Performance of Machine Repair Problem with Balking, Reneging, Warm spares and Synchronous Multiple Vacation
}

\author{
Mingjian $\mathrm{Ma}^{1, *}$ and Bing Zhao ${ }^{2}$ \\ ${ }^{1}$ Office of Academic Affairs, University of Yan Shan , Hebei 066004, China \\ ${ }^{2}$ College of Science, University of Yan Shan, Hebei 066004, China
}

\begin{abstract}
In this paper, we studied the machine repair model with impatient behaviors-balking and reneging. There are several warm spares and repairmen who take the synchronous multiple vacations in the system. We use the method of blocked matrix to solve the steady-state probability equations. And we acquired some obvious iterative expressions of steady-state performances, such as the availability, failure frequency and the mean time to first failure of the system. Moreover, we carried out some numerical analysis and investigated the effect of parameters on the performance measures about reliability.
\end{abstract}

Keywords-failure frequency; the mean time to first failure; balk; renege; reliability

\section{INTRODUCTION}

There have been so many researches paying attention to the machine repair problem for a long time. The machine repair problem has been broadly exerted to solve the problems in machine operating system, communication system , production line system and so on. In many papers, authors consider the machine repair model as the queueing model with finite capacity. Gradually, researchers added reneging, balking and vacation into the model which made the machine repair system more feasible in realistic situations.

In the machine repair system, they may join the queue waiting for repairing when some units failed. In practice, there are some impatient units which may not join the queueing and another part of them may leave the queue without being repaired. The behaviors above are known as balking and reneging, which is often appeared in real life situations. These behaviors have been considered in many studies. But most of the sudies only deal with queueing theory in the viewpoint of the system, rather than some reliability and availability problem of the system. Shawky investigated the $M / M / 1 / \mathrm{m} / \mathrm{m}$ machine interference model with balking in [1], reneging and single server, while there is another server joining the system for longer queues. He derived iterative formula of the steadystate probability and some special cases for both the $M / M / 1 / m / m$ model and the $M / M / 2 / m / m$ model. Shawky developed the machine interference model in [2], and he studied $M / M / C / K / N$ model with balking, reneging and spares. Ke and Wang analyzed the $M / M / R$ machine repair model with balking, reneging, and breakdown of server in [3]. they gained the cost analysis at last. Wang investigated the machine repairable system with balking in [4], reneging and warm standbys. This paper presented some reliability characteristics such as steady-state availability, the mean time to first failure(MTTFF) of the system and some numerical analysis.

In real situation, when there are no failed units in the system on the service time, the repairmen may take some subsidiary tasks in the term of cost for free time. This random time is called "vacation" in the queueing systems. Vacation policy has been developed in the machine interference by many researchers. Gupta studied the machine repair problem with warm spares, and the server take vacations providing exhaustive service in [5]. He deduced the steady-state probability distribution as well as some results involving queueing problems. The machine repair system with reneging, warm spares and the repairman following N-policy discipline was analyzed by Jain in [6]. Some reliability performances and numerical results were obtained.

However, the machine repair problem combining balking, reneging, and vacation policy was seldom studied by researchers in aspect of reliability theory. Yanping Sun and Dequan Yue studied $M^{X} / M / 1 / N$ queueing system with batch arrival, balking, reneging and multiple vacation in [7]. They developed the multi-server queueing systems with balking, reneging and vacation in [8]. Some performances measures in the viewpoint of queueing theory were deduced in $[7,8]$. Recently, Dequan Yue, Wuyi Yue and Hongjuan Qi invertigated a machine repair model with warm spares and two heterogeneous repairmen in [9]. The authors studied the model both from the viewpoint of queueing and reliability. They also developed a cost model obtaining the optimization analysis.

This paper deals with one machine repair model incorporating balking, reneging, warm spares and the synchronous multiple vacation in the viewpoint of reliability and availability. The method of blocked matrix algorithm was used to derive stead-state availability, failure frequency and the mean time to first failure of the system. At last, this paper performed a parametric analysis which provides numerical results to show the effects of parameters on the index of the model.

\section{THE MODEL ASSUMPTIONS}

The designed model is based on the following assumptions. 
- $\quad$ There are $m$ operating units and $w$ warm spares in the system $(m+w=k)$ and the lifetime distributions of which is exponentially distributed respective with rates $\lambda$ and $\alpha$.

- There are $R$ repairmen, and the repair time is exponentially distributed with rates $\mu$. If there are no failed units, the repairmen go to vacation. The vacation time is exponentially distributed with rates $\theta$. The repairmen serve according to FIFO discipline.

- The impatient failed units balk with probability 1-b, while join the queue with $b$.

- The failed unit waiting in the queue may depart without being repaired and the waiting time is exponentially distributed with rates $r$

- As soon as there are failed units in the system, they may be replaced by a new unit at once and sent for repairing immediately. If the repairing of the failed units is finished, the units may be as good as new ones.

- $\quad$ All of the parameters are respectively independence.

\section{PERFORMANCE ANALYSIS}

\section{A. Steady-state Probability Equations}

Let $N(t)$ and $J(t)$ respectively be the number of the failed units and the state of the repairman in the system at time $t$, $J(t)=\left\{\begin{array}{l}1, \text { (The server is on the service at the time } t) \\ 0 \text {, (The server is on vacation at the time } t \text { ) }\end{array}\right.$

then, $\{N(t), J(t), t \geq 0\}$ is a Makov process with state space as following:

$$
\Omega=\{(0,0)\} \cup\{(i, j) \mid i=1,2, \mathrm{~L}, k ; j=0,1\}
$$

Define the state space of unit on working as following:

$$
W=\{(0,0),(1,0),(1,1), \mathrm{L},(k-1,0),(k-1,1)\}
$$

and define the state space of unit failed as following:

$$
\begin{gathered}
F=\{(k, 0),(k, 1)\} \\
\pi_{00}=p_{00}=\lim _{t \rightarrow \infty}\{N(t)=0, J(t)=0\} \\
\pi_{i, j}=p_{i, j}=\lim _{t \rightarrow \infty}\{N(t)=i, J(t)=j\}, \quad i=1,2, \mathrm{~L}, k ; j=0,1
\end{gathered}
$$

Then, according to the Makov process theory, we get the steady-state probability equations as following:

$$
\begin{gathered}
\lambda_{0} b \pi_{00}=r \pi_{10}+\mu \pi_{11}, i=0, j=0 \\
\left(\lambda_{i} b+i r+\theta\right) \pi_{i 0}=\lambda_{i-1} b \pi_{i-1,0}+(i+1) r \pi_{i+1,0}, \\
i=1,2, \mathrm{~L}, k-1, j=0 \\
(k r+\theta) \pi_{k 0}=\lambda_{k-1} b \pi_{k-1,0}, i=k, j=0
\end{gathered}
$$

$$
\begin{gathered}
\left(\lambda_{1}+\mu\right) \pi_{11}=\theta \pi_{10}+2 \mu \pi_{21}, i=1, j=1 \\
\left(\lambda_{i}+i \mu\right) \pi_{i 1}=\theta \pi_{i 0}+\lambda_{i-1} \pi_{i-1,1}+(i+1) \mu \pi_{i+1,1}, \\
i=2, \mathrm{~L}, R-1, j=1 \\
\left(\lambda_{R} b+R \mu\right) \pi_{R 1}=\theta \pi_{R 0}+\lambda_{R-1} \pi_{R-1,1}+(R \mu+r) \pi_{R+1,1} \\
i=R, j=1 \\
{\left[\lambda_{i} b+R \mu+(i-R) r\right] \pi_{i 1}=\theta \pi_{i 0}+\lambda_{i-1} b \pi_{i-1,1}+[R \mu+(i+1-R) r) \pi_{i+1,1},} \\
i=R+1, \mathrm{~L}, k-1, j=1 \\
{[R \mu+(k-R) r] \pi_{k 1}=\theta \pi_{k 0}+\lambda_{k-1} b \pi_{k-1,1}, i=k, j=1}
\end{gathered}
$$

The steady-state probability equations above can be expressed in the matrix form as following:

$$
\boldsymbol{\pi} \boldsymbol{A}=\boldsymbol{0}
$$

where $\boldsymbol{\pi}=\left\{\pi_{00}, \pi_{10}, \pi_{11}, \mathrm{~L}, \pi_{k 0}, \pi_{k 1}\right\}$

The normalizing condition is:

$$
\sum_{i=0}^{k} \pi_{i 0}+\sum_{i=1}^{k} \pi_{i 1}=1
$$

The transition rate matrix $\mathbf{A}$ of the Markov process has the following blocked matrix structure:

$$
\boldsymbol{A}=\left(\begin{array}{ccccc}
\boldsymbol{A}_{0} & \boldsymbol{C}_{0} & & & \\
\boldsymbol{B}_{1} & \boldsymbol{A}_{1} & \boldsymbol{C}_{1} & & \\
& \mathrm{O} & \mathrm{O} & \mathrm{O} & \\
& & \boldsymbol{B}_{k-1} & \boldsymbol{A}_{k-1} & \boldsymbol{C}_{k-1} \\
& & & \boldsymbol{B}_{k} & \boldsymbol{A}_{k}
\end{array}\right)
$$

where the subsidiary matrices are expressed as following:

$$
\begin{gathered}
\boldsymbol{A}_{0}=-\lambda_{0} b, \boldsymbol{B}_{1}=(r, \mu)^{T}, \boldsymbol{C}_{0}=\left(\lambda_{0} b, 0\right) \\
\boldsymbol{A}_{i}=\left(\begin{array}{cc}
a_{i 1} & \theta \\
0 & a_{i 2}
\end{array}\right), 1 \leq i \leq k \\
a_{i 1}=-\left(\lambda_{i} b+i r+\theta\right), a_{i 2}=-\left(\lambda_{i}+i \mu\right), 1 \leq i \leq R-1 \\
a_{i 1}=-\left(\lambda_{i} b+i r+\theta\right), a_{i 2}=-\left[\lambda_{i} b+R \mu+(i-R) r\right], \\
R \leq i \leq k-1 \\
a_{k 1}=-(k r+\theta), a_{k 2}=-[R \mu+(k-R) r] \\
\boldsymbol{B}_{i}=\left(\begin{array}{cc}
b_{i 1} & 0 \\
0 & b_{i 2}
\end{array}\right), 2 \leq i \leq k \\
b_{i 1}=i r, b_{i 2}=i \mu, 2 \leq i \leq R \\
b_{i 1}=i r, b_{i 2}=R \mu+(i-R) r, R+1 \leq i \leq k
\end{gathered}
$$




$$
\begin{gathered}
C_{i}=\left(\begin{array}{cc}
c_{i 1} & 0 \\
0 & c_{i 2}
\end{array}\right), 1 \leq i \leq k-1 \\
c_{i 1}=\lambda_{i} b, c_{i 2}=\lambda_{i}, 1 \leq i \leq R-1 \\
c_{i 1}=\lambda_{i} b, c_{i 2}=\lambda_{i} b, R \leq i \leq k-1
\end{gathered}
$$

Otherwise, the mean failure rate of the system is represented as follow:

case1: $w<R$

$$
\lambda_{i}= \begin{cases}m \lambda+(w-i) \alpha, & i=0, \mathrm{~L}, \quad w-1 \\ (m+w-i) \lambda, & i=w, \mathrm{~L}, R-1 \\ (m+w-i) \lambda b, & i=R, \mathrm{~L}, m+w-1 \\ 0, \quad \text { others } & \end{cases}
$$

case2: $w \geq R$

$$
\lambda_{i}=\left\{\begin{array}{l}
m \lambda+(w-i) \alpha, i=0, \mathrm{~L}, \quad R-1 \\
{[m \lambda+(w-i) \alpha] b, \quad i=R, \mathrm{~L}, w} \\
(m+w-i) \lambda b, \quad i=w+1, \mathrm{~L}, m+w-1 \\
0, \quad \text { others }
\end{array}\right.
$$

\section{B. Matrix-geometric Solution}

In this part, we use the method of blocked matrix to obtain the obvious iterative expression of the steady-state probability vectors.

Theorem Define $\boldsymbol{\pi}=\left(\boldsymbol{\pi}_{0}, \boldsymbol{\pi}_{1} \mathrm{~L} \boldsymbol{\pi}_{i}\right)$ be the steady-state probability vector of the transition rate matrix $\mathbf{A}$, where $\boldsymbol{\pi}_{0}=\left(\pi_{00}\right), \boldsymbol{\pi}_{i}=\left(\pi_{i 0}, \pi_{i 1}\right),(i=1,2, \mathrm{~L}, k)$.

The steady-state probability (1) above can be rewritten in the matrix form as:

$$
\left\{\begin{array}{l}
\boldsymbol{\pi}_{0} \boldsymbol{A}_{0}+\boldsymbol{\pi}_{1} \boldsymbol{B}_{1}=0 \\
\boldsymbol{\pi}_{i} \boldsymbol{C}_{i}+\boldsymbol{\pi}_{i+1} \boldsymbol{A}_{i+1}+\boldsymbol{\pi}_{i+2} \boldsymbol{B}_{i+2}=0 \\
\boldsymbol{\pi}_{k-1} \boldsymbol{C}_{k-1}+\boldsymbol{\pi}_{k} \boldsymbol{A}_{k}=0 \quad, \quad i=0,1, \mathrm{~L}, k-2
\end{array}\right.
$$

then $\boldsymbol{\pi}_{i}$ is represented iteratively as following:

$$
\boldsymbol{\pi}_{i}=\boldsymbol{\pi}_{k} \boldsymbol{T}_{i} \quad i=0,1, \mathrm{~L}, k-1
$$

where

$$
\begin{gathered}
\boldsymbol{T}_{0}=-\boldsymbol{T}_{1} \boldsymbol{B}_{1} \boldsymbol{A}_{0}^{-1} \\
\boldsymbol{T}_{k-1}=-\boldsymbol{A}_{k} \boldsymbol{C}_{k-1}^{-1},
\end{gathered}
$$

$$
\begin{gathered}
\boldsymbol{T}_{k-2}=-\left(\boldsymbol{B}_{k}+\boldsymbol{T}_{k-1} \boldsymbol{A}_{k-1}\right) \boldsymbol{C}_{k-2}^{-1} \\
\boldsymbol{T}_{i}=-\left(\boldsymbol{T}_{i+2} \boldsymbol{A}_{i+2}+\boldsymbol{T}_{i+1} \boldsymbol{B}_{i+1}\right) \boldsymbol{C}_{i}^{-1}, \quad i=1, \mathrm{~L}, k-3
\end{gathered}
$$

Proof It can be seen obviously that $\left|A_{0}\right| \neq 0$ and $\left|C_{i}\right| \neq 0(\mathrm{i}=1, \mathrm{~L} \mathrm{k}-1)$. From the last equation of (2), we get:

$$
\boldsymbol{\pi}_{k-1}=-\boldsymbol{\pi}_{k} \boldsymbol{A}_{k} \boldsymbol{C}_{k-1}^{-1}=\boldsymbol{\pi}_{k} \boldsymbol{T}_{k-1}
$$

where $\boldsymbol{T}_{k-1}=-\boldsymbol{A}_{k} \boldsymbol{C}_{k-1}^{-1}$

Equation (8) is substituted into (2) in which $i=k-2$ to yield:

$$
\boldsymbol{\pi}_{k-2}=-\boldsymbol{\pi}_{k}\left(\boldsymbol{B}_{k}+\boldsymbol{T}_{k-1} \boldsymbol{A}_{k-1}\right) \boldsymbol{C}_{k-2}^{-1}=\boldsymbol{\pi}_{k} \boldsymbol{T}_{k-2}
$$

where $\boldsymbol{T}_{k-2}=-\left(\boldsymbol{B}_{k}+\boldsymbol{T}_{k-1} \boldsymbol{A}_{k-1}\right) \boldsymbol{C}_{k-2}^{-1}$

We can deduce the rest through substituting the results obtained successively into (2), where $i=k-3, k-4, \mathrm{~L}, 1$. Thus, (3) and (7) is deduced.

Moreover, the result is substituted into the first equation of (2) to yield:

$$
\boldsymbol{\pi}_{0}=-\boldsymbol{\pi}_{k} \boldsymbol{T}_{1} \boldsymbol{B}_{1} \boldsymbol{A}_{0}^{-1}=\boldsymbol{\pi}_{k} \boldsymbol{T}_{0}
$$

At last, we obtain the equations as following:

$$
\left\{\begin{array}{l}
\pi_{0}+\sum_{i=1}^{k} \pi_{i} \boldsymbol{e}_{2}=1 \\
\boldsymbol{\pi}_{0} \boldsymbol{C}_{0}+\boldsymbol{\pi}_{1} \boldsymbol{A}_{1}+\boldsymbol{\pi}_{2} \boldsymbol{B}_{2}=0
\end{array}\right.
$$

Equation (3) is substituted into (10) to yield:

$$
\left\{\begin{array}{l}
\boldsymbol{\pi}_{0}+\boldsymbol{\pi}_{k} \sum_{i=1}^{k} \boldsymbol{T}_{i} \boldsymbol{e}_{2}=1 \\
\boldsymbol{\pi}_{k}\left(\boldsymbol{T}_{0} \boldsymbol{C}_{0}+\boldsymbol{T}_{1} \boldsymbol{A}_{1}+\boldsymbol{T}_{2} \boldsymbol{B}_{2}\right)=0
\end{array}\right.
$$

where $\boldsymbol{e}_{2}$ is a column vector with two components, and each component of $\boldsymbol{e}_{2}$ equal to one. The steady-state probability vector $\boldsymbol{\pi}_{k}$ can be deduced from (11), and $\boldsymbol{\pi}_{i}$ ( $i=1$, 2, L $k-1$ ) can be expressed according to (3).

\section{Performance Measures of System}

Corollary The steady-state availability of the system is given by: 


$$
A=\sum_{j \in W} \boldsymbol{\pi}_{j} \boldsymbol{e}=\boldsymbol{\pi}_{0}+\sum_{j=1}^{k-1} \boldsymbol{\pi}_{j} \boldsymbol{e}_{2}=\boldsymbol{\pi}_{k}\left(\boldsymbol{T}_{0}+\sum_{j=1}^{k-1} \boldsymbol{T}_{j} \boldsymbol{e}_{2}\right)=1-\boldsymbol{\pi}_{k} \boldsymbol{e}_{2}
$$

The steady-state failure frequency of the system is given by:

$$
M=\boldsymbol{\pi}_{k-1} \boldsymbol{C}_{k-1} \boldsymbol{e}_{2}=\lambda_{k-1} b\left(\pi_{k-1,0}+\pi_{k-1,1}\right)
$$

Proof According to Theorem 1 and the theory which can be referred in [10],the inferences above are easily obtained.

Theorem The formula of the mean time to first failure of the system is known as following:

$$
\text { MTTFF }=\int_{0}^{+\infty} R(t) d t=\lim _{s \rightarrow 0} R^{*}(s)=-\boldsymbol{Q}_{W}(0) \boldsymbol{B}^{-1} \boldsymbol{e}_{W}
$$

where $\boldsymbol{e}_{W}$ is a column vector with $\boldsymbol{W}$ components, and each component of $\boldsymbol{e}_{W}$ equal to one. The matrix $\boldsymbol{B}$ is obtained from matrix $\boldsymbol{A}$, in which the last two rows and the last two columns for the absorbing states are deleted.

$$
\text { Define } \quad\left(\boldsymbol{x}_{0}, \mathrm{~L} \quad \boldsymbol{x}_{k-1}\right) \boldsymbol{B}=-\boldsymbol{Q}_{W}(0),
$$

where $\quad \boldsymbol{x}_{0}=\left(x_{00}\right), \boldsymbol{x}_{1}=\left(x_{10}, x_{11}\right), \mathrm{L}, \boldsymbol{x}_{k-1}=\left(x_{k-1,0}, x_{k-1,1}\right)$, $\boldsymbol{Q}_{W}(0)=(0,1,0, \mathrm{~L}, 0)$.

Equation (12) can be expressed in the matrix form as:

$$
\left\{\begin{array}{l}
\boldsymbol{x}_{0} A_{0}+\boldsymbol{x}_{1} \boldsymbol{B}_{1}=0 \\
\boldsymbol{x}_{0} \boldsymbol{C}_{0}+\boldsymbol{x}_{1} \boldsymbol{A}_{1}+\boldsymbol{x}_{2} \boldsymbol{B}_{2}=-(1,0) \\
\boldsymbol{x}_{i} \boldsymbol{C}_{i}+\boldsymbol{x}_{i+1} A_{i+1}+\boldsymbol{x}_{i+2} \boldsymbol{B}_{i+2}=0, i=1, \mathrm{~L} k-3 \\
\boldsymbol{x}_{k-2} \boldsymbol{C}_{k-2}+\boldsymbol{x}_{k-1} \boldsymbol{A}_{k-1}=0
\end{array}\right.
$$

The MTTFF can be expressed as following:

$$
M T T F F=\boldsymbol{x}_{0}+\boldsymbol{x}_{1} \boldsymbol{e}_{2}+\mathrm{L}+\boldsymbol{x}_{k-1} \boldsymbol{e}_{2}
$$

where

$$
\begin{gathered}
\boldsymbol{x}_{i}=\boldsymbol{x}_{k-1} \boldsymbol{H}_{i}, \quad i=0,1, \mathrm{~L} k-2 \\
\boldsymbol{H}_{0}=-\boldsymbol{H}_{1} \boldsymbol{B}_{1} \boldsymbol{A}_{0}^{-1} \\
\boldsymbol{H}_{i}=-\left(\boldsymbol{H}_{i+1} \boldsymbol{A}_{i+1}+\boldsymbol{H}_{i+2} \boldsymbol{B}_{i+2}\right) \boldsymbol{C}_{i}^{-1}, i=1, \mathrm{~L} k-4 \\
\boldsymbol{H}_{k-3}=-\left(\boldsymbol{H}_{k-2} \boldsymbol{A}_{k-2}+\boldsymbol{B}_{k-1}\right) \boldsymbol{C}_{k-3}^{-1} \\
\boldsymbol{H}_{k-2}=-\boldsymbol{A}_{k-1} \boldsymbol{C}_{k-2}^{-1} \\
\boldsymbol{x}_{k-1}=-(1,0)\left(\boldsymbol{H}_{0} \boldsymbol{C}_{0}+\boldsymbol{H}_{1} \boldsymbol{A}_{1}+\boldsymbol{H}_{2} \boldsymbol{B}_{2}\right)^{-1}
\end{gathered}
$$

Proof From the last equation of (13)

$$
\begin{gathered}
\boldsymbol{x}_{k-2}=-\boldsymbol{x}_{k-1} \boldsymbol{A}_{k-1} \boldsymbol{C}_{k-2}^{-1}=-\boldsymbol{x}_{k-1} \boldsymbol{H}_{k-2} \\
\text { where } \boldsymbol{H}_{k-2}=-\boldsymbol{A}_{k-1} \boldsymbol{C}_{k-2}^{-1}
\end{gathered}
$$

Substituting the result (18) into (13) where $i=k-3$, we get:

$$
\boldsymbol{x}_{k-3}=-\boldsymbol{x}_{k-1}\left(\boldsymbol{H}_{k-2} \boldsymbol{A}_{k-2}+\boldsymbol{B}_{k-1}\right) \boldsymbol{C}_{k-3}^{-1}=-\boldsymbol{x}_{k-1} \boldsymbol{H}_{k-3}
$$

where $\boldsymbol{H}_{k-2}=-\boldsymbol{A}_{k-1} \boldsymbol{C}_{k-2}^{-1}$

Substituting the results obtained successively into the previous one of (13), we get:

$$
\begin{gathered}
\boldsymbol{x}_{i}=-\boldsymbol{x}_{k-1}\left(\boldsymbol{H}_{i+1} \boldsymbol{A}_{i+1}+\boldsymbol{H}_{i+2} \boldsymbol{B}_{i+2}\right) \boldsymbol{C}_{i}^{-1}=\boldsymbol{x}_{k-1} \boldsymbol{H}_{i}, \\
i=1, \mathrm{~L}, k-4 \\
\boldsymbol{x}_{0}=-\boldsymbol{x}_{k-1} \boldsymbol{H}_{1} \boldsymbol{B}_{1} \boldsymbol{A}_{0}^{-1}=\boldsymbol{x}_{k-1} \boldsymbol{H}_{0} .
\end{gathered}
$$

Substituting (14) into the second equation of (13), we get:

$$
\boldsymbol{x}_{k-1}=-(1,0)\left(\boldsymbol{H}_{0} \boldsymbol{C}_{0}+\boldsymbol{H}_{1} \boldsymbol{A}_{1}+\boldsymbol{H}_{2} \boldsymbol{B}_{2}\right)^{-1}
$$

Thus, $\boldsymbol{x}_{0}, \boldsymbol{x}_{1}$, L $\boldsymbol{x}_{k-2}$ are expressed by $\boldsymbol{x}_{\boldsymbol{k}-\boldsymbol{1}}$, and the mean time to first failure of the system is derived as following:

$$
\begin{gathered}
\text { MTTFF }=\boldsymbol{x}_{0} \boldsymbol{e}_{2}+\mathrm{L}+\boldsymbol{x}_{k-1} \boldsymbol{e}_{2}=\boldsymbol{x}_{k-1} \boldsymbol{H}_{0}+\boldsymbol{x}_{k-1}\left(\boldsymbol{H}_{1}+\mathrm{L}+\boldsymbol{H}_{k-1}\right) \boldsymbol{e}_{2} \\
=-(1,0)\left(\boldsymbol{H}_{0} \boldsymbol{C}_{0}+\boldsymbol{H}_{1} \boldsymbol{A}_{1}+\boldsymbol{H}_{2} \boldsymbol{B}_{2}\right)^{-1}\left[\boldsymbol{H}_{0}+\left(\boldsymbol{H}_{1}+\mathrm{L}+\boldsymbol{H}_{k-1}\right) \boldsymbol{e}_{2}\right]
\end{gathered}
$$

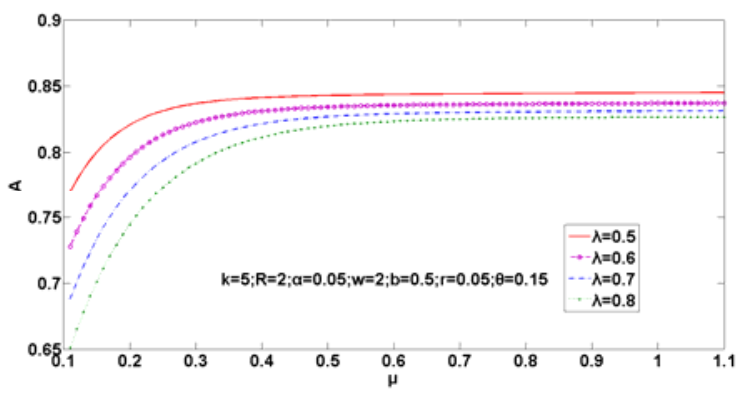

FIGURE I. EFFECT OF $\lambda$ ON $\alpha$

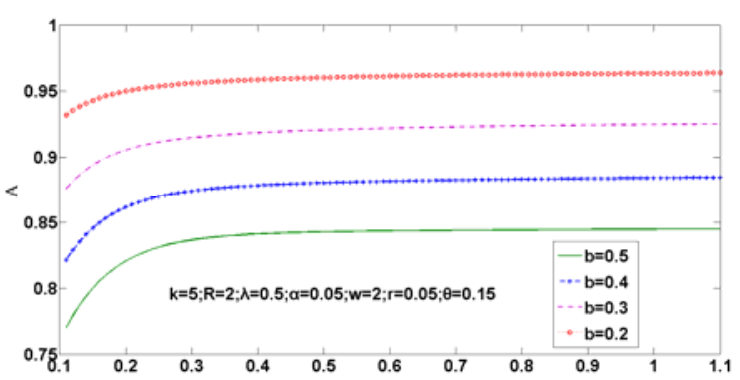

FIGURE II. EFFECT OF $\beta$ ON $\alpha$ 


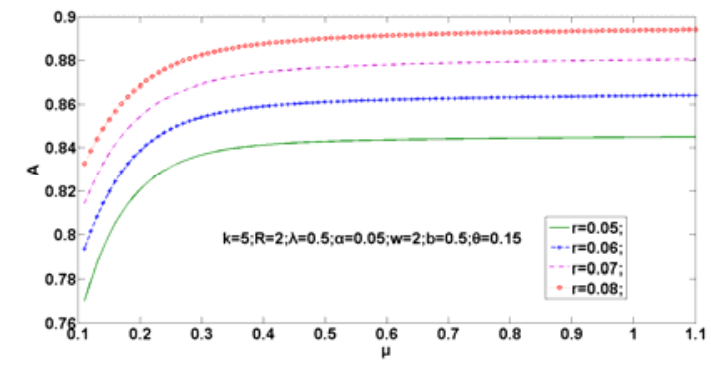

FIGURE III. EFFECT OF r ON $\alpha$

\section{NUMERICAL EXAMPLES}

We provide the numerical results of the steady-state availability, and the mean time to first failure of the system. Firstly, the effects of parameters on the steady-state availability of $A$ are presented.

Figures 1-3 show that the steady-state availability $A$ increases respectively as failure rate $\lambda$ decreases. However, the steady-state availability $A$ increases respectively as the increasing of $\mu, b, \theta$ or $r$. Also it can be seen that the steadystate availability $A$ increases remarkably as $\mu$ varies from 0.1 to 0.3 , and indistinctively while $\mu$ is higher than 0.3 .

It is described from Figures 1-2 that the influences of $\lambda$ or $b$ on $A$ is significant, and theinfluence of $r$ on Figures 3 is weak.

Next, the effects of various system parameters on the mean time to first failure MTTFF are investigated.

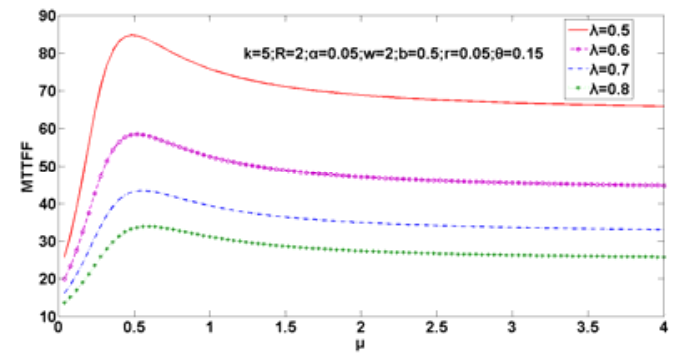

FIGURE IV. EFFECT OF $\lambda$ ON MTTFF

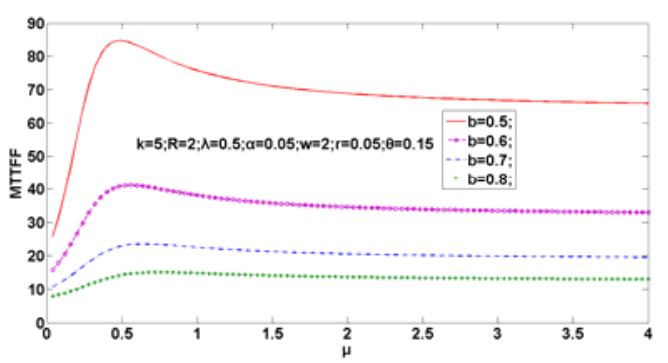

FIGURE V. EFFECT OF b ON MTTFF

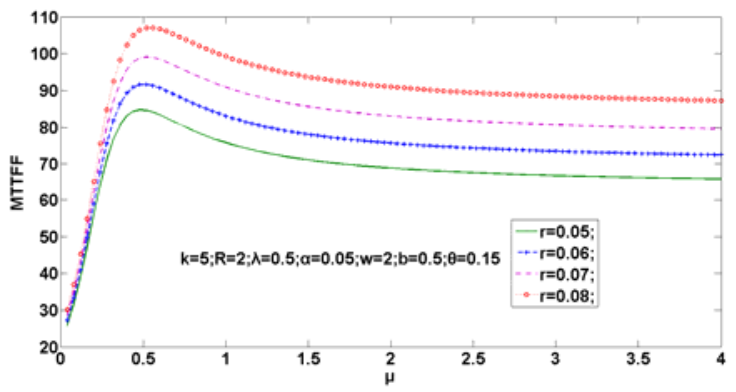

FIGURE VI. EFFECT OF r ON MTTFF

It can be observed from Figures 4-6 that MTTFF increases significantly as repair rate $\mu$ increases, while the value of $\mu$ varies from 0 to 0.5 . However MTTFF decreases unsignificantly as $\mu$ increasing higher than 0.5 .

In Figures 4 , we set $k=5, R=2, \alpha=0.05, w=2$, $b=0.5, r=0.05, \theta=0.15$, and $\lambda$ vary from 0.5 to 0.8 . In Figures 5 , we set $k=5, R=2, \lambda=0.5, \alpha=0.05, w=2, \theta=$ 0.15 , and vary $b$ from 0.5 to 0.8 . We can see from Figures 5-6 that MTTFF decreases drastically as $\lambda$ or $b$ increases for small $\lambda$ and small $b$, respectively. However, the rate of decrease in MTTFF is not significant for large $\lambda$ and large $b$.

In Figures 6, we set $k=5, R=2, \lambda=0.5, \alpha=0.05, w=$ $2, b=0.5, \theta=0.15$ and vary reneging rate r from 0.05 to 0.08 . It can be seen that MTTFF increases as reneging rate $r$ increases.

\section{CONCLUSIONS}

In this paper, the machine repair model with balking, reneging, warm spares and repairmen taking the synchronous multiple vacations was analyzed. We derived succinct expressions of some performance measures of the system in the viewpoint of reliability by using the method of blocked matrix. The numerical analysis was carried out to presenting the effect of parameters on steady-state availability and the mean time to first failure of system. Based on the numerical analysis, we can optimize the system model which was built up according to the practical problems.

\section{ACKNOWLEDGMENT}

This work is supported by the National Youth Natural Science Foundation of China (No. 11301458).

\section{REFERENCES}

[1] A. Shawky. "The Single-server Machine Interference Model with Balking, Reneging and an Additional Server for Longer Queues”. Microelectronics Reliability, no. 2, vol. 37, pp. 335-357, 1997,.

[2] A. Shawky, "The Machine Interference Model: $M / M / C / K / N$ with Balking Reneging and Spare”, OPSEARCH, no. 1, vol. 36, pp. 25-35, 2003

[3] J. Ke, K. Wang. "Cost Analysis of the M/M/r Machine Repair Problem with Balking, Reneging and Server Breakdowns". Journal of the Operational Research Society, vol. 50, pp. 275-282, 2005 
[4] K. Wang, J. Ke. "Probability analysis of a repairable system with warm standbys plus balking and reneging”, Applied Mathematical Modelling, vol. 27, pp. 327-336, 2003.

[5] S. Gupta, "Machine interference problem with warm spares, server vacations and exhaustive service”, Performance Evaluation, no. 3, vol. 29, pp. 195-211, 1997.

[6] M. Jain, Rakhee, S. Maheshwari, "N-policy for a machine repair system with spares and reneging”, Applied Mathematical Modelling, no. 6, vol. 28, pp. 513-531, 2004.

[7] Y. Sun, D. Yue, "Performance analysis of $M^{X} / M / 1 / N$ Queuing System with Balking,Reneging, Batch Arrival and Multiple Vacations”, Operations Research and Management Science, no. 6, vol. 15, pp. 60-65, 2006.

[8] D. Yue, Y. Sun, "Waiting Time of $M / M / c / N$ Queuing System with Balking, Reneging, and Multiple Synchronous Vacations of Partial Servers”, Systems Engineering, no. 2, vol. 28, pp. 89-97, 2008.

[9] D. Yue, W. Yue , Hongjuan Qi, "Performance analysis and optimazation of a machine repair problem with warm spares and two heterogeneous repairmen”, Optim Eng, vol. 13, pp. 545-562, 2012.

[10] J. Cao, K. Cheng, Introduction to Mathematical Reliability, Beijing: Higher Education Press, 2006, pp. 193-197. 